\title{
Professionenmix in der
}

\section{Patientenversorgung: \\ ein weiterer steiniger Weg}

Die Debatte um Rollenverteilungen und Zuständigkeiten in der medizinischen Versorgung hat vehement begonnen. Die in diesem Zusammenhang am häufigsten auftauchenden Begriffe lauten „Ärztemangel“ und „Delegation ärztlicher Leistungen". Damit bleibt die Diskussion einstweilen oft noch der Idee verhaftet, man könne die gewohnten Kooperationsformen der Fachberufe im Gesundheitswesen im wesentlichen beibehalten. Das Krankheitenpanorama, und nicht allein der viel beschworene Kostendruck, erfordert aber grundlegendere Veränderungen in der Beschreibung von Kompetenzen und Kooperationen für eine angemessene Versorgung. Ein hierfür geeignetes Schlagwort lautet „Professionenmix". Das damit skizzierte Aufgabenpaket wiegt schwer.

\section{Norbert Schmacke}

Die Umgangssprache ist häufig sehr aussagekräftig. „Fragen Sie Ihren Arzt oder Apotheker" deutet auf zwei Schlüsselberufe hin, wobei der zweite vom Rezept des ersten lebt. Ärztliche Dominanz zeichnet das Gesundheitswesen aller Länder aus, wobei es unterschiedliche Varianten der ärztlichen Hegemonie gibt. Ärztinnen und Ärzte in den reichen Industrienationen sehen das häufig anders: sie befürchten, dass sie ihre Machtposition schon in erheblichem Maße eingebüßt haben. In Deutschland wird seit einigen Jahren diskutiert, ob ein befürchteter Ärztemangel in strukturschwachen Regionen durch Aufwertung der früheren Gemeindeschwester kompensiert werden kann. Scheinbar über Nacht ist die Rede von der Delegation ärztlicher Leistungen vor allem auf die Pflege. Der Sachverständigenrat hat schon 2007 deutlich gefordert, dass Deutschland durch eine Neufassung des Professionenmix den Anschluss an internationale Entwicklungen finden möge; tatsächlich ist die Entschlossenheit, mit der von der deutschen Ärzteschaft das Diagnose- und Behandlungsmonopol reklamiert wird, erstaunlich stark ausgeprägt. Nachfolgend soll gezeigt werden, dass die Diskussion um eine Neuverteilung der Rollen unterschiedlicher Fachberufe des Gesundheitswesen noch weitaus stärker als bisher einge-

Prof. Dr. med. Norbert Schmacke ist Hochschullehrer für Gesundheitswissenschaften an der Universität Bremen und leitet die Arbeits- und Koordinierungsstelle Gesundheitsversorgungsforschung. Er ist stellvertretendes unparteiisches Mitglied im Gemeinsamen Bundesausschuss. bunden werden sollte in die Frage, ob die aus bestimmten Blickwinkeln ja fraglos auch attraktiv erscheinende Aufstellung des jetzigen Versorgungssystems in Deutschland zukunftsweisend ist, oder ob nicht doch die innewohnenden Strukturschwächen zu lange kleingeredet worden sind und damit Zeit zur Entwicklung eines bedarfsgerechteren Systems vertan worden ist. Anders formuliert: ob in der Diskussion zugelassen wird, quer zu denken und sich von manchen liebgewordenen aber ineffizienten Lösungen zu verabschieden. Der Text intendiert nicht, die z.B. vom Sachverständigenrat empirisch hinterlegte Argumentation noch einmal zu wiederholen sondern mittels zugespitzter Thesen Phantasie freizusetzen, wie an die Stelle ermüdender Endlosdebatten der Mut zur modellhaften Erprobung neuer Wege treten könnte. Dazu sind einige gedankliche Ausflüge erforderlich, ehe das Thema des Professionenmix am Ende bilanzierend ins Blickfeld genommen wird. Die Eingangsthesen lauten:

- Die Katastrophenberichte über drohenden Ärztemangel lenken vor der Frage ab, wie in Zeiten erschwerter ökonomischer Rahmenbedingungen und einer absehbaren Mangelsituation in einer Vielzahl von qualifizierten Berufen in neuer Weise Standards für eine angemessene medizinische-pflegerische Versorgung entwickelt werden können. Wenn die gewohnten Besitzstände der Ärzteschaft in gut bis überversorgten Regionen zum Maßstab genommen werden, wird es keine Lösungen für eine faire Verteilung der ärztlichen Kompetenzen über das Land geben können. 


\section{THEMA}

- Die Szenarien einer unabwendbaren Kostenexplosion durch demographischen Wandel und medizinischen Fortschritt lenken von der Frage ab, was unter relevantem medizinischen Fortschritt künftig verstanden werden soll und wo gleichzeitig der Medikalisierung der Alltagswelten und der sozialen Konflikte Einhalt geboten werden muss.

- Die an Fahrt gewinnende Abwicklung des Solidaritätsprinzips in der gesetzlichen Krankenversicherung versperrt den Blick darauf, dass sämtliche "grundlegenden“ Alternativmodelle der Finanzierung von Versorgungssystemen bezüglich Kostenbremsung und Qualitätssteigerung ernüchternd bis desaströs ausgefallen sind. Es fragt sich, wie eine parlamentarische Mehrheit dafür gewonnen werden kann, eine einheitliche Bürgerversicherung durchzusetzen, die diesen Namen auch verdient. Dass hierfür relativ lange Zeiträume erforderlich sind, könnte fast die Entschlossenheit zur Einschlagung dieses Weges bremsen.

- Gerade bei einem alles in allem hohen medizinischen Versorgungsniveau muss lauter als bisher die Frage gestellt werden, wie Ressourcenverschwendung wirksam eingedämmt werden kann. Dabei wäre - naiv gesprochen - ein Preis für eine gute Idee auszusetzen, wie Ärztinnen und Ärzte dazu veranlasst werden können, Stolz in einer möglichst genauen Indikationsstellung zu entwickeln. Niemand glaubt im Ernst daran, dass die enormen Verordnungsmengen im Arzneimittelbereich etwas mit realem Bedarf zu tun haben. Eine der großen Schwächen der Versorgungsforschung liegt wohl darin, dass es sehr schwer ist, Primärdaten im Alltag zu erheben, salopp formuliert: in die Küche zu blicken.

Nun stellt sich die Frage, warum die bisherigen Reformbemühungen um eine Modernisierung der Versorgungsstrukturen so außerordentlich schleppend verlaufen. Es hat ja nicht an guten Ideen gefehlt, selbst nicht an klugen Reformimpulsen im alles entscheidenden (?) Sozialgesetzbuch. Eigentlich müsste es doch längst einen lebhaften Wettbewerb um die besten Versorgungsmodelle geben, begleitet von der Gewinnung wichtiger Daten zu Qualität und Wirtschaftlichkeit. Vielleicht ist der Mut aber doch nicht groß genug. Was damit gemeint ist, soll in mehreren Schritten wiederum thesenartig vorgestellt werden:

Es ist an der Zeit, über die Weiterentwicklung des deutschen Hausarztsystems nachzudenken. Dies wirkt auf den ersten Blick vielleicht als Überforderung, ist es doch gerade erst gelungen, die jahrzehntelange unerträgliche Diskriminierung der Hausarztmedizin in Ansätzen zu überwinden. Und trotzdem zeigt gerade die problematische Situation in strukturschwachen Regionen besonders gut, dass das Modell des allein oder vielleicht auch in Doppelpraxis arbeitenden Hausarztes allein nicht mehr für alle Anforderungen trägt. Einer der Gründe dafür ist, dass jüngere Ärztinnen und Ärzte der drohenden Vereinzelung skeptisch gegenüberstehen. Ein weiterer Grund ist, dass eine annähernd flächendeckende gleichmäßige Versorgung gerade der Vielzahl älterer, multimorbider Menschen andere Konzepte verlangt.
Und dies gilt nicht nur für strukturschwache Regionen. Derartige Neuansätze müssen erprobt und evaluiert werden, anders formuliert: in solche Reformen muss investiert werden, sie lassen sich nicht am runden Tisch erfinden und ergeben sich auch nicht spontan durch den von vielen herbeigesehnten Wettbewerb. Diese Einschätzung, zugleich Forderung, wird hier zum ersten Mal formuliert; sie taucht im weiteren Verlauf noch mehrfach auf. Und es fragt sich, wie Mehrheiten dafür gewonnen werden können, Forschung und Entwicklung auf der Strukturebene für genauso wichtig zu halten wie auf der Ebene von individueller Diagnostik und Therapie. Es kommt dabei auch darauf an, den fundamentalen Unterschied zwischen einer legitimatorischen Begleitevaluation und einer methodisch gut gemachten Begleitforschung zur Ermittlung der Überlegenheit neuer Versorgungsmodelle gegenüber der Regelversorgung deutlich zu machen. Wie viel davon in einem wettbewerblich organisierten System besser zu entwickeln ist, das ist eine weitere ernsthafter zu diskutierende Frage.

- In der gesundheitspolitischen Debatte wird berechtigterweise auf die deutsche Spezialität der doppelten Facharztschiene hingewiesen. Es gibt aber ein zweites Problem, das selbst bei einer (zur Zeit völlig utopischen) Reduzierung von fragwürdiger Dopplung bestehen bliebe. Und das betrifft die Frage, wie die spezialistische Medizin sinnvoller mit der „Basisversorgung“ verzahnt werden kann. Gemeint ist, dass der klassische Weg der Überweisung zu Spezialisten und die Entgegennahme eines Konsiliarberichtes die Diskussion um den für die jeweiligen Patientinnen und Patienten sinnvollen Wege nicht ersetzen können. Für derartige Kommunikation ist aber, wenn man in der bisherigen Logik bleibt, „keine Zeit". Die Frage ist, wie lange sich derart komplexe System es sich leisten können, „keine Zeit“ zu rufen, wenn Abstimmungsprozesse unverzichtbar sind. Geändert werden muss m.a.W. nicht allein etwas an der doppelten Facharztschiene, sondern zugleich an dem weithin unvermittelten Nebeneinander von allgemeinmedizinischer und spezialistischer Diagnostik, Beratung und Therapie. Hierfür gibt es leider auch keine Modelle aus der Schublade. Gesundheitsökonomisch hat sich der Capitation-Ansatz mit dieser Problematik am ehesten zukunftsweisend beschäftigt.

- Moderne Versorgungssysteme werden sich ganz anders als heute mit der Frage beschäftigen müssen, wie das Wissensmanagement für die Professionellen wie die ratsuchenden und kranken „Laien“ organisiert werden kann. Das noch nicht breit akzeptierte Megathema der nächsten Jahrzehnte heißt „Health Literacy“. Weder Therapeutinnen und Therapeuten noch Patientinnen und Patienten sind ohne intelligente, die best verfügbare Evidenz aufbereitende Informations- und Beratungssysteme in der Lage, die Spreu vom Weizen zu trennen und à jour zu sein, wenn es Not tut. Diese Forderung ist insofern „ver-rückt“, als sie tatsächlich das bisherige Verständnis von Professionen und Laientum auf den Kopf stellt. Es geht dabei wohl bemerkt nicht um die Verwischung der Rollen von Professionellen und Laien, 
sondern um die Frage der Verfügbarkeit relevanten aktuellen Wissens bei der Suche nach angemessenen diagnostischen und therapeutischen Lösungen. Dies bedeutet am Ende die Verabschiedung von dem klassischen Verständnis, dass Professionen über ein nicht zu hinterfragendes Vorratswissen verfügen, das automatisch vertrauensbildend wirkt. Das Thema „Health Literacy“ ist die Fortführung der Diskurse um die evidenzbasierte Medizin und die Risikokommunikation und beinhaltet insofern mehr als die Frage nach reinen Sachinformationen. Ein ermutigender Ansatz in diese Richtung im Bereich Herzkreislauf-Erkrankungen ist das internetbasierte ARRIBA als Informationsgrundlage zur Erörterung von Behandlungsoptionen bei erhöhtem cardiovaskulärem Risiko; bislang aber fast ein Solitär in der Landschaft. Es ist nicht in Stein gemeißelt, dass solche innovativen Beratungskonzepte unter das Arztprivileg fallen.

- Die Themen Verkürzung stationärer Verweildauern, Behandlungsoptionen wie Multimorbidität und Zunahme hochbetagter Menschen führen geradezu zwangsläufig zum Ansatz des Fallmanagement. Es wird sich zeigen, dass auch hier das Argument „keine Zeit“ kontraproduktiv ist. Zeit für kompetentes Fallmanagement muss Teil der Versorgungskonzepte werden. Es fragt sich auch hier, wann Mehrheiten dafür gewonnen werden können, diesen Ansatz gründlicher zu pilotieren und zu evaluieren. Dabei geht es letztlich um einen Befreiungsschlag aus dem Argumentationszirkel „Wir würden als Ärzte ja gern ausführlicher beraten, wenn wir mehr Zeit hätten und dafür angemessen honoriert würden“. Patentlösungen für dieses Aufgabenspektrum gibt es nun aber auch wiederum nicht, so dass ein weiteres Mal die Forderung erhoben wird: es muss in die Entwicklung solcher vorbildlicher Modelle investiert werden. Es stellt sich dabei nicht zuletzt die Frage, ob wirklich der Hausarzt bisherigen Typs der geborene Fallmanager ist (das wird heute in der Reformpolitik ja weithin angenommen) oder ob er nicht zumindest der massiven Unterstützung durch andere Berufsgruppen bedarf, am Ende vielleicht gar ein neues Berufsbild entsteht?

- Überfällig ist weiter Forschung zu telemedizinischen Ansätzen in der Primärversorgung. Die heutigen Abwehrgefechte großer Teile der Ärzteschaft wirken kurios. Aber es fragt sich natürlich sehr wohl, für welche Einsätze „Tele“ bzw. allgemeiner die elektronische Erfassung relevanter Daten den größten Nutzen verspricht. Es liegt auf der Hand, dass die Arzneimittelsicherheit gezielt erhöht werden kann, wenn es gelingt, problematische Interaktionen und unsinnige Mehrfachverschreibungen auf diesem Weg systematisch zu vermeiden. Die zentrale Zukunftsfrage wird lauten: welche Modelle der elektronischen Patientenakte setzen sich unter dem Aspekt Sicherheit und Geschwindigkeit durch? Elektronisch gestützte Formate bieten insgesamt völlig neue Chancen in der Kommunikation zwischen den Fachberufen, Institutionen wie zwischen Professionellen und Patienten.
Innerhalb der Professionendebatte hat die Frage „Delegation oder Substitution“ inzwischen einen festen Platz erobert. Die zugehörigen Thesen lauten:

- Die größte Denkblockade entsteht wahrscheinlich durch das Haften an den jetzigen Mengengerüsten des Leistungsgeschehens. Solange es nur darum geht, für die Aufrechterhaltung des Status Quo „Ersatzlösungen“ zu suchen, fehlt jede Bereitschaft zu visionären Konzepten.

- Sprachlich finden die momentanen gedanklichen Verkürzungen ihren Ausdruck im Begriff des Ärztemangels. Dieser Begriff ist nicht einmal für die extrem strukturschwachen Regionen hilfreich, weil er unterschlägt, dass die Aufrechterhaltung einer hohen Lebensqualität dort ja nicht allein dadurch erschwert wird, dass Vertragsarztsitze nicht in gewohnter Form wiederbesetzt werden können.

- Im Medizinsystem selber wird dabei die Frage vernachlässigt, wie es um das Thema Pflegemangel aussieht: auch die Pflege wird vor große Herausforderungen gestellt, den Bedarf im ambulanten und stationären Sektor sicherzustellen und zugleich ihre Potenziale auszubauen. Wenn jetzt schnell von Übernahme ärztlicher Leistungen durch andere Fachberufe, vor allem die Pflege, die Rede ist, dann ist das zum einen folgerichtig, weil dies in vielen anderen Ländern Realität ist. Es darf zum anderen aber nicht verkannt werden, dass Pflege und Medizin traditionell unterschiedlichen Handlungslogiken folgen und beide sich für die Übernahme eines neuen Rollenverständnisses rüsten müssen. Dabei spielt der extreme Statusunterschied sicher eine gewichtige Rolle; dies verweist auf die Notwendigkeit der Weiterführung der Akademisierung nichtärztlicher Fachberufe.

- Die bisherige Forschung belegt sehr wohl, dass entsprechend qualifizierte Pflegekräfte bei gleicher Qualität in erheblichem Umfang ,ärztliche“ Leistungen übernehmen können. Der in der Politik weithin erhoffte Einspareffekt lässt sich demgegenüber bisher nicht überzeugend nachweisen.

- Mit Blick auf die Nachwuchsprobleme in der Allgemeinmedizin stellt sich die Frage, ob die ja gerade erst wieder stärker werdende Identität dieser Arztgruppe sich durch die Debatte um den Professionenmix irritieren lässt oder aber in ihr Chancen sieht, die viel beschworene Funktion des Gatekeepers und/oder Koordinators im Leistungsgeschehens besser als heute wahrnehmen zu können. Bislang setzen Hausärzte ja sehr stark auf die Funktionserweiterung der Medizinischen Fachangestellten und meinen, auf die Erweiterung ihrer Teams durch Pflegekräfte verzichten zu können. Das ist vermutlich eine bedeutsame Fehleinschätzung, die nicht nur ökonomischen Kalküls folgt sondern auch etwas mit der Furcht vor Konkurrenz im Bereich der Vertrauensbindung mit den Patientinnen und Patienten zu tun haben dürfte. Insofern ist zu begrüßen, dass innerhalb der Deutschen Gesellschaft für Allgemeinmedizin die Übertragbarkeit des Chronic-Care-Modells von Ed Wagner ein zentrales Thema geworden ist. Diesem Modell ist die 


\section{THEMA}

vorurteilsfreie Suche nach einem optimalen Professionenmix zugehörig.

Gender ist für die Medizin und das Versorgungssystem immer noch ein Fremdbegriff, die Bedeutung der Frage einer für Männer wie Frauen auf der Seite der Professionellen wie der Patienten angemessenen Strukturierung der Praxis wird erst in Ansätzen begriffen. Thesen hierzu lauten:

- Für bestimmte Bereichen wie die Altenpflege wird es quasi als selbstverständlich angesehen, dass hier der Frauenanteil bei 85 Prozent liegt, während auf der Leitungsebene 60 Prozent Männer zu finden sind. Weder die Einkommenssituation noch Hierarchiefragen noch die Verbleibeprobleme erhalten angemessene öffentliche Aufmerksamkeit.

- Während Pflege generell immer als weibliche „Domäne" betrachtet wurde und wird, entwickelt sich nun die Ärzteschaft Schritt für Schritt zu einer stark von Frauen besetzten Berufsgruppe. Auch hier finden sich wie in der Pflege Hierarchie- und Karriereprobleme. Erst allmählich setzt das Nachdenken darüber ein, wie das große Potenzial der Ärztinnen entfaltet und dauerhaft genutzt werden kann.

- Angesichts der Veränderung der Alterskohorten ist absehbar, dass der unmittelbare Versorgungsbereich der Medizin und Pflege sich mit der Konkurrenz anderer attraktiver Beschäftigungsfelder in der Gesellschaft auseinandersetzten muss. Frauen wie Männer für die Arbeit im Gesundheitswesen zu interessieren und sie im Beruf zu halten, wird ein Schlüsselkompetenz des Managements werden - sollte eigentlich heute schon der Fall sein.

- Das Thema Work-Life-Balance ist ganz offenkundig kein Modethema sondern wird gerade im Zusammenhang mit dem Ringen um qualifizierte Mitarbeiterinnen und Mitarbeiter eine zunehmende Bedeutung erlangen. Das heißt, dass sowohl im ambulanten wie im stationären Sektor die Gestaltung akzeptierter Arbeitsbedingungen für Einzelne wie für Paare unverzichtbar wird. $\mathrm{Ob}$ dies ein fördernder Faktor für die patientengerechte Weiterentwicklung von Organisationsformen sein wird, ist schwer prognostizerbar.

Das Thema Migration ist im Gesundheitswesen noch nicht richtig angekommen. Hier zeigen sich Zeitverzögerungen wie in den meisten anderen gesellschaftlichen Bereichen auch. Thesen hierzu lauten:

- Migrantinnen und Migranten sind ein unausgeschöpftes Potenzial für das Gesundheitswesen. Das gilt sowohl für bereits Beschäftigte, die besser gefördert werden könnten, als auch für Arbeitssuchende und neue $\mathrm{Zu}$ wanderer.

- Sprachliche und fachliche Förderung müssen insoweit selbstverständliche Elemente der Weiterentwicklung der Gesundheitsberufe werden. Von derartigen Anstrengungen profitieren auch „deutsche“ Frauen und Männer, weil damit eine Professionalisierung der gesamten Erwachsenenbildung verbunden sein wird.
- Die Finanzierung dieser Aktivitäten wird bisherige Budgets für Aus-, Fort- und Weiterbildung sprengen und erfordert neue Aushandlungsprozesse.

Eine erste Zwischenbilanz lautet: Die Realisierung neuer Versorgungsmodelle unter Einschluss eines deutlich geänderten Professionenmix hängt nicht nur von der Beachtung ökonomischer Faktoren ab, sondern ebenso von mentalen und psychosozialen Faktoren. Die Einsicht in die Veränderung gewohnter Strukturen und Kooperationsformen muss gefördert werden. So wird noch das Potenzial qualifizierter Frauen für diese Prozesse vermutlich - überwiegend unwissend - verspielt. Der Konkurrenzkampf um qualifizierte Arbeitskräfte allgemein wird härter werden. Systeminnovationen werden auch Investitionen erfordern, die nicht als „,add on“ gedacht werden dürfen, sondern in Gesamtbudgets einzukalkulieren sind. Es gibt zudem für keine der bisher angesprochenen Probleme „gesetzte Erfolge“, so dass zumindest größere Reformschritte und Pilotvorhaben sorgfältig evaluiert werden müssen. In einem wirklich extrem arztzentrierten und vermachteten System sind derartige Visionen schwer durchzusetzen. Zudem werden radikale Fragen, die dringend gestellt werden müssen, stets auf massive Abwehr stoßen. Dies soll exemplarisch verdeutlicht werden:

- Die Operationshäufigkeit in der Gynäkologie könnte deutlich weiter gesenkt werden. Verschreibungsmengen für weibliche Hormone bewegen sich immer noch in kritischen Bereichen. Der jährliche Rhythmus der Früherkennungsuntersuchungen auf Gebärmutterhalskrebs könnte tendenziell auf einen Drei-Jahres-Rhythmus umgestellt werden; die meisten Abstriche können von „Nicht-Ärztinnen“ entnommen werden. Die hohen Kaiserschnittraten könnten mit Entängstigungsstrategien und ökonomischen Dis-Incentives deutlich gesenkt werden. Das alles hätte zur Folge, dass die Zahl niedergelassener und operierender Gynäkologinnen und Gynäkologen deutlich verringert werden könnte. Man muss sehen, dass im ambulanten Sektor die hohe Kontaktdichte im frauenärztlichen Bereich gerade von den Wiederholungsrezepten und den Abstrichuntersuchungen getrieben wird.

- Gilt das nur für dieses Fachgebiet? Natürlich nicht. Warum verschreiben in Deutschland automatisch Ärzte Brillen? Warum sehen Orthopäden immer noch so viele Menschen mit unkomplizierten Rückenschmerzen? Warum sehen auch Rheumatologen so viele Menschen mit unkomplizierten Gelenkbeschwerden? Warum werden so viele Typ-2-Diabetiker von Diabetologen behandelt? Warum haben Kardiologen das Schieben von Herzkathetern zu einer scheinbaren Bagatelluntersuchung entwickeln können? Was ist der Beitrag der Allergologen im Umgang mit dem Massenphänomen „Allergien“?

- Und wie sieht es im hausärztlichen Bereich aus? Hier plädieren inzwischen immer mehr Ärztinnen und Ärzte selber für eine Reduktion der Kontaktdichte und eine Konzentration auf Kernaufgaben. Das wird aber nur funktionieren, wenn die Organisation der Praxisabläufe geändert wird und tatsächlich ein neuer Professionen- 
mix in die Praxen einzieht. Dies war und ist das Kernanliegen des Chronic Care Modells von Ed Wagner. Erst dann wird auch die Erwartung realisierbar werden, dass der Hausarzt bzw. die allgemeinmedizinische Praxis fachlich zum Lotsen im System wird. Dabei werden unterschiedliche Größen und Formen nötig sein, nicht zuletzt in Abhängigkeit von den Regionen. Die Honorare werden auf mehr Schultern zu verteilen sein.

Entwicklungen im skizzierten Sinn setzen voraus, Patientinnen und Patienten und deren Interessenvertreter zu beteiligen. Patientenzufriedenheit, wenn sie denn gut gemessen wird, kann zu einer Schlüsselkategorie des Prozesses werden. Versicherte wie Patienten können bei allen Etappen von Reformprozessen eingebunden werden, auch bei der Entwicklung von Forschungsdesigns und der Bewertung von Forschungsergebnissen. Das führt noch einmal zur Rolle der Forschung:

- Leider wissen wir zu den Potenzialen „nicht-ärztlicher“ Fachberufe bei der Neudefinition der Rollen zu wenig, um neue Versorgungsmodelle einfach im Baukastenverfahren zusammensetzen zu können. Es bedarf unterschiedlicher Modelle, die zwingend evaluiert werden müssen, und zwar bezüglich relevanter klinischer wie ökonomischer Endpunkte. Das ist alles andere als trivial und geht über das Verständnis der klassischen Evaluationen von Modellvorhaben des SGB V deutlich hinaus. Ob diese Erkenntnis bei den anstehenden Modellen nach § 63 c SGB V Berücksichtigung finden wird, ist fraglich. Immerhin wäre dies eine große Chance.

- Ohne derartige hochwertige Studien wird es aber extrem schwer sein, Delegations- wie Substitutionsansätze im Sinne eines grundlegend neuen Professionenmix durchzusetzen. Aus wissenschaftlicher Sicht ist aber auch noch einmal festzuhalten, dass es keine Blaupausen aus anderen Ländern gibt, die wir in Deutschland nur umsetzten müssten.
- Gelingt es nicht, für derartige anspruchsvolle Versorgungsforschung und Modelle Mittel einzuwerben, bleibt es beim „Trial and Error" und vor allem der Logik des "Löcherstopfens“. Man wird sicher auch auf pragmatische Modellvorhaben angewiesen bleiben, wenn es gilt, Durststrecken zu überwinden. Klug wäre es aber allemal, mittel- und langfristig in Forschung zu Systeminnovationen zu investieren. Wenn diese Einsicht vorhanden ist, wird sich leichter die Frage nach den Verantwortlichkeiten klären lassen. Man sollte meinen, dass die medizinischen Fakultäten eine Vorbildfunktion ausüben; die Hoffnung ist beim Autor aber schwach ausgeprägt. Das Bundesforschungsministerium hat sich demgegenüber in den letzten Jahren, auch im Zusammenwirken mit den Kassen, als relativ mutig erweisen. Die Deutsche Forschungsgemeinschaft beginnt, Fragen der Versorgungsforschung auch für die Grundlagenforschung zu entdecken. Die bisher über diese Kanäle zur Verfügung gestellten Mittel reichen aber für die hier angesprochenen Entwicklungen im Bereich der Weiterbildung von Professionen und deren Kooperation nicht aus. Und es ist auch klar, dass am Ende der Erfolg dieser Bemühungen davon abhängt, ob es gelingt, das Verhältnis von Leistungsmengen und Qualität nachvollziehbarer und transparenter zu gestalten, um dies diplomatisch auszudrücken. Wie immer bei großen Reformen kann es nicht nur Gewinner geben.

Letzter Satz: es kommt wohl vor allem darauf an, die Einsicht zu fördern, dass tatsächlich der Patient im Mittelpunkt der Überlegungen zu stehen hat. Dann wird auch deutlich werden, dass es nicht sehr sinnvoll ist, vom Ärztemangel zu sprechen, sondern Indikatoren dafür zu entwickeln, wie man eine annähernd gleiche und akzeptable Versorgungsqualität zwischen den Regionen dieser Republik entwickeln kann. Die Neuverteilung der Rollen der Fachberufe spielt dabei eine entscheidende Rolle.

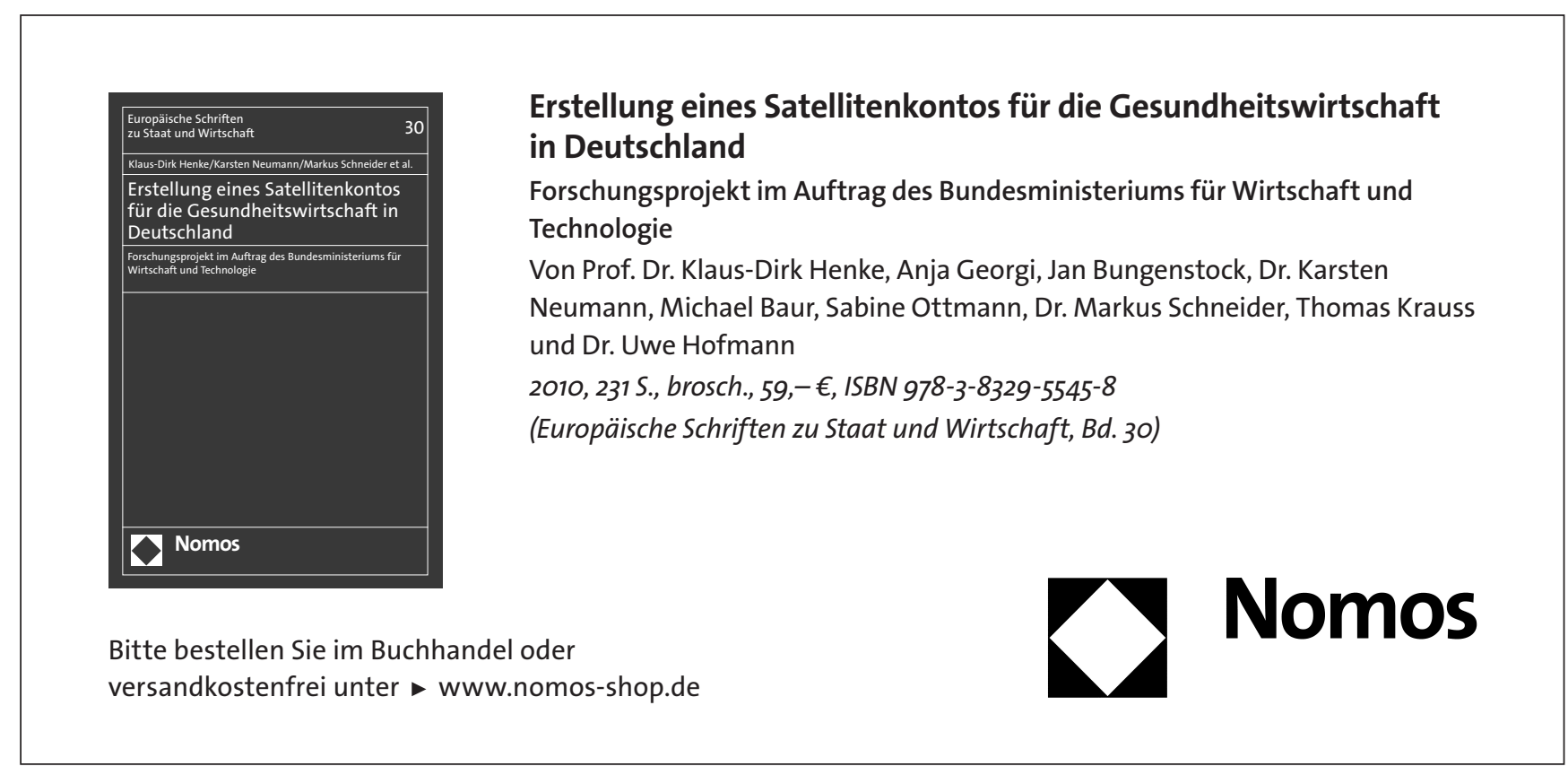

